Article type: Original research

Title: Gravitational anti-screening and predictions within the solar system

Author: $\quad$ A. Raymond Penner

Address: Department of Physics, Vancouver Island University, 900 Fifth Street,

Nanaimo, BC, Canada

V9R 5S5

Email: $\quad$ raymond.penner@ viu.ca

Tel: $\quad 250753-3245$ ext:2336 


\title{
Gravitational Anti-screening and Predictions within the Solar System
}

\begin{abstract}
The theory of gravitational anti-screening, which has previously been applied to various astronomical observations as an alternative to dark matter, is now applied to the solar system. The effect that gravitational anti-screening has on the precession rate of the planets will be determined and compared to current observations. In addition, the effect that gravitational anti-screening will have on certain orbital parameters of long-period comets will be determined. The set of 119 long-period comets that are considered in this paper have a broad peak of aphelion distances, based on current gravitational theory, centred at approximately $50 \mathrm{kAU}$. In the case of gravitational antiscreening, these aphelion distances will be shown to be greatly reduced to a narrow distribution centred at approximately $10 \mathrm{kAU}$. It is further found from the theory that comets orbiting at this distance will have orbital speeds approximately $20 \%$ greater than that predicted from Newtonian gravitational theory.
\end{abstract}

\section{Introduction}

The theory of gravitational anti-screening is an alternative to the theory of dark matter. Its initial virtue was that it naturally led to the Baryonic Tully-Fisher Relationship (BTFR). The BTFR is the cornerstone of alternate theories to dark matter such as gravitational anti-screening and MOND (MOdified Newtonian Dynamics). The BTFR is a relationship between $\mathrm{M}_{\mathrm{B}}$, the baryonic mass of a galaxy, and $\mathrm{v}_{\mathrm{R}}$, the surprisingly, nearly constant orbital speed of the stars in a galaxy's outer reaches. The relationship as given by McGaugh (2000) is

$$
\mathrm{M}_{\mathrm{B}}=\mathrm{A} \mathrm{v}_{\mathrm{R}}{ }^{4}
$$

where

$$
\mathrm{A}=(47 \pm 6) \mathrm{M}_{\odot} \mathrm{km}^{-4} \mathrm{~s}^{4}
$$

This relationship indicates that in the outer regions of a galaxy the enclosed galactic mass is directly proportional to the distance to the galactic centre. The BTFR does not fit naturally with the theory of dark matter. For proponents of alternate theories, the BTFR is the Achilles heel of dark matter. 
The theory of gravitational anti-screening (Penner 2016a, 2018) is based on a model of the screening of electric charges by virtual electric dipoles that is found in QED. In the case of QED, this screening results in the value of the observed charge being much less than that of the bare charge. Applying the same model to gravity leads to anti-screening and the BTFR. This antiscreening results in the value of the observed mass being much greater than that of the bare mass. The screening of electric charge as found in QED and the larger observed masses of galaxies and galactic clusters would therefore appear to be two sides of the same coin.

It is not just that the theory of gravitational anti-screening leads naturally to the BTFR. In previous work, the theory of gravitational anti-screening has been applied to the rotational curve of the Galaxy, to the rotational curves of spiral galaxies in general, and to the dynamics of the Coma cluster (Penner 2016a, 2018). The theoretical results were found to be in very good agreement with the corresponding astronomical observations. The theory has also been applied to binary galaxies (Penner $(2017,2018)$ where it is shown that there is a relationship between the line-of-sight velocity difference of the pair and the individual rotational velocities of the galaxies. The theory is found to be in excellent agreement with the observations taken by multiple researchers for the case of the binaries being on radial orbits. In addition, the theory was applied to the structure of the universe itself (Penner 2016b, 2018). Using a model of the distribution of superclusters, the overall density parameter of the universe, as determined by the theory, is $\Omega=$ $1.08 \pm 0.19$ consistent with a geometrically flat universe. In addition, the energy density which falls out from the theory has a negative pressure associated with it, allowing for agreement with the accelerated expansion of the universe that is currently attributed to dark energy.

Given these excellent results, with agreement found over such a wide range of astronomical observations, the question arises as to why the theory of gravitational anti-screening has been largely ignored. A primary reason is that the theory relies on the existence of mass dipoles, typically taken to be composed of two bound particles. There have been various models of mass dipoles presented over the years and theories using such mass dipoles to explain astronomical observations currently attributed to dark matter. In these theories the cosmos is taken to be filled with these mass dipoles, either real or virtual, which by some mechanism align with the gravitational field. In these models of mass dipoles, one of the two particles has its inertia mass, passive gravitational mass, and active gravitational mass all positive. The differences between the models is with respect to the second particle. The simplest case is where the second particle has a 
positive inertial mass but negative active and passive gravitational masses (Blanchet 2007a, Hajdukovic 2011a-c, 2012a-b). Such a mass dipole will align in a gravitational field analogous to an electric dipole in an electric field. Unfortunately, a particle that has its inertial and passive gravitational masses of opposite signs violates the equivalence principle. A model which avoids this is one where the second particles inertial and passive gravitational masses are positive but whose active gravitational mass is negative (Blanchet 2007b, Blanchet \& Le Tiec 2008). Although such a dipole does not violate the equivalence principle, having a particle or dipole whose active gravitational mass is not equal to its passive gravitational mass does violate the law of action and reaction. Also such a dipole will not naturally align in a gravitational field.

A dipole model presented by Bondi (1957) overcomes both problems by having all of the masses, including the inertial mass, of the second particle negative. Such a dipole will have a very strange behaviour, in that if the two particles are bound non-gravitationally the dipole will on its own accelerate along the axis joining the two particles in the direction of the negative mass. The dipole moment is therefore anti-parallel to the particle's velocity vector. Indeed, although it is strange, Bondi has shown that this behaviour is allowed in GR as long as the magnitude of the positive mass exceeds that of the negative mass, if only slightly, and if such dipoles come in pairs of opposite orientation. These conditions allow one to define a centre of mass reference frame for the system. Although, such mass dipoles will obey the equivalence principle and will free fall in a gravitational field like any particle, by having each of the component particles with their passive gravitational mass and inertial masses equal, the mass dipoles will again not naturally align in a gravitational field.

This manuscript will first provide a new model of mass dipoles to be used in the theory of gravitational anti-screening. Using the theory, the mass density distribution due to the mass dipoles along with the resulting gravitational field surrounding an isolated solar mass will then be determined. It will be found that the BTFR applies even to a single solar mass. The significant effect that the external galactic field has on the results will then be included. Following this, the effect that the resulting larger gravitational field has on orbiting bodies within our solar system will be considered. This includes determining the precession rate of orbiting bodies and the effect on the orbiting parameters of long-period comets. Currently, the limits on any possible deviations from current gravitational theory within our solar system are provided by the analysis of the ephemerides of solar system bodies. These observations lead to fairly severe constraints with 
regards to the precession rate of Saturn's orbit. From positional observations of planets and spacecraft, Pitjev \& Petjeva (2013), have determined that the residual precession rate for Saturn is

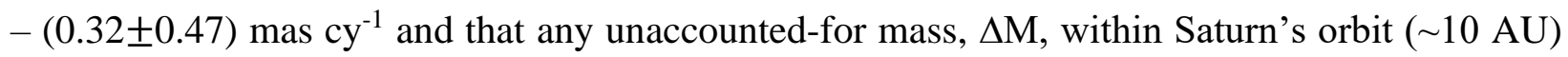
must be $<1.7$ x $10^{-10} \mathrm{M} \odot$. Using radio tracking data of the Cassini spacecraft, Hees et al (2014) constrained the precession rate of Saturn to $(0.43 \pm 0.43)$ mas $\mathrm{cy}^{-1}$. This determination was based on a constraint on the quadrupole moment which arises in certain versions of MOND.

\section{Theory}

\subsection{Mass dipole model}

Instead of a mass dipole which involves a bound pair of particles, the author proposes the existence of a single massless particle which has an intrinsic mass dipole moment. Like Bondi's dipoles, such particles will obey the equivalence principle, and, like Bondi's dipoles, they will have their dipole moment anti-parallel to their velocity vector. The massless particle will not have a defined centre of mass frame, i.e. like a photon, there is no reference frame for which the particle will be at rest. Although such a single massless particle will solve the problems associated with mass dipoles and particles with a negative mass, it will, again like Bondi's dipoles, by obeying the equivalence principle not naturally align in a gravitational field. The only interaction that the hypothesized particle will have with other particles is gravitational and that only via its mass dipole moment.

The concept of a point particle with an intrinsic mass dipole moment may seem strange, but within the Standard model it is predicted that an electron, a point particle itself, has an intrinsic electric dipole moment which is aligned with its spin. In the case of the electron, the contribution that this intrinsic electric dipole moment makes to the electron's overall electric field is negligible, while for the hypothesized particle its intrinsic mass dipole moment is the source of its gravitational field.

\subsection{Gravitational anti-screening}

In the theory of gravitational anti-screening, a given mass is taken to be the source, albeit indirectly, of the point mass dipoles described above. The mechanism of how this takes place is as follows. The given mass, via its gravitational interactions with other masses, will be surrounded by a field of virtual gravitons. These virtual gravitons in turn are taken to have a probability of 
dissociating into virtual mass dipoles which are taken to come into existence with their velocity vector pointing away from the given mass and thereby with their mass dipole moment aligned with the gravitational field. This is unlike other proposed theories where the cosmos is taken to be filled with mass dipoles which then align in a gravitational field. The situation is analogous to QED where virtual photons, surrounding a given charge, have a probability of dissociating into virtual electric dipoles. In QED these virtual temporal electric dipoles come into existence with their electric dipole moment anti-parallel with the electric field. The result is that these virtual electric dipoles cause a screening of the electric charge which greatly reduces the value of the observed charge. In the case of gravitational anti-screening, the temporal virtual mass dipoles are coming into existence with their mass dipole moment parallel with the gravitational field. The result is an anti-screening effect which greatly increases the value of the observed mass. Although the theory may currently lack a strong theoretical basis, the conceptual symmetry that it has with QED is attractive.

Analogous to the classical electromagnetic case for a dielectric, the equivalent mass density $\rho_{\mathrm{v}}$, due to a given mass dipole distribution, is determined by

$$
\rho_{\mathrm{V}}=-\nabla \cdot \mathbf{P}_{\mathbf{G}}
$$

where $\mathbf{P}_{\mathbf{G}}$ is the mass dipole moment density surrounding a given baryonic mass. The resulting gravitational field $\mathbf{g v}$ at a distance $\mathrm{r}$ from the given mass, due to the mass dipole distribution, will therefore be given by

$$
\mathbf{g v}_{\mathbf{V}}=\frac{\mathrm{G}}{\mathrm{r}^{2}} \int_{\mathrm{V}} \rho_{\mathrm{V}} \hat{\mathbf{r}} \mathrm{dV}
$$

Equations (2) and (3) are the gravitational equivalent of the contribution that electric dipoles in a dielectric make to the electric field in electromagnetics. The net gravitational field surrounding a baryonic mass will thus be given by

$$
\mathbf{g}=\mathbf{g}_{\mathbf{B}}+\mathbf{g}_{\mathbf{v}}
$$

where $\mathbf{g}_{\mathbf{B}}$ is the gravitational field due to the baryonic mass itself. It is important to note that the mass density as given by (2) is a real mass density surrounding a given baryonic mass and the theory in this sense is akin to the theory of dark matter.

It may be expected that the value of $\mathbf{P}_{\mathbf{G}}$, the mass dipole moment density, would be directly proportional to $\mathbf{g}$, the total gravitational field. However, in the case of spherical symmetry, having PG being directly proportional to $\mathbf{g}$ leads to a $\rho_{\mathrm{v}}$ having a value of zero outside of the baryonic 
mass and therefore cannot lead to the BTFR. To determine the required dependence that $\mathbf{P}_{\mathbf{G}}$ needs to have on $\mathbf{g}$, in order for the model to lead to the BTFR, the dependence that $\mathbf{P}_{\mathbf{G}}$ has on $\mathbf{g}$ will first be expressed by

$$
\mathbf{P}_{\mathbf{G}}=-\frac{1}{4 \pi \mathrm{G}} f(\mathrm{~g}) \mathbf{g}
$$

where $f(\mathrm{~g})$ is a dimensionless function of $\mathrm{g}$. It is surmised that fundamentally $f(\mathrm{~g})$ relates to the dependence that the probability of a graviton dissociating into a mass dipole has on the local gravitational field.

In the case of spherical symmetry, it follows from (2), (3), and (5) that

$$
\mathrm{g}_{\mathrm{V}}=\mathrm{g} f(\mathrm{~g}) \text {. }
$$

In the outer reaches of galaxies, where $g \rightarrow 0$, the field due to the dipole distribution must be significantly greater than the field due directly to the baryonic mass, i.e. $g \rightarrow g_{V}$. It then follows that in the weak field limit the function $f(\mathrm{~g}) \rightarrow 1$. All that is then required for the relationship, as given by (5), to lead to agreement with the BTFR is that in the weak field limit, as $g \rightarrow 0$, the function $f(\mathrm{~g})$ can be expanded as

$$
f(\mathrm{~g})=1-\alpha\left(\frac{\mathrm{g}}{\mathrm{g}_{0}}\right) \pm \beta\left(\frac{\mathrm{g}}{\mathrm{g}_{0}}\right)^{2} \pm \ldots
$$

where $g_{0}$ is a parameter to be determined from observations and $\alpha$ and $\beta$ are constants which will depend on the specific function chosen. It follows that, for the case where $\mathrm{g} \ll<\mathrm{g}_{0}$, the total gravitational field surrounding a baryonic mass of mass $\mathrm{M}_{\mathrm{B}}$ will to a good approximation, by (4), (6), and (7), be given by

$$
\mathrm{g} \cong \mathrm{g}_{\mathrm{B}}+\mathrm{g}-\alpha\left(\frac{\mathrm{g}}{\mathrm{g}_{0}}\right) \mathrm{g} .
$$

Therefore by (8), in this weak field limit,

$$
\mathrm{g}=\left(\frac{\mathrm{g}_{0} \mathrm{~g}_{\mathrm{B}}}{\alpha}\right)^{1 / 2}
$$

Equation (9), with the substitutions of $g=v^{2} r^{-1}$ and $g_{B}=G M_{B} r^{-2}$, then leads to

$$
\mathrm{M}_{\mathrm{B}}=\frac{\alpha}{\mathrm{Gg}_{0}} \mathrm{v}^{4},
$$

namely the BTFR. Equating the coefficients of (1) and (10) results in

$$
\mathrm{g}_{0}=\alpha\left(1.6 \times 10^{-10} \mathrm{~m} \mathrm{~s}^{-2}\right) \text {. }
$$

Many functions will of course fit the requirement as given by (7). In this sense, the theory of gravitational anti-screening is akin to MOND where a moderately constrained free function 
leads to the BTFR. In previous work several functions for $f(\mathrm{~g})$ have been used. In Penner (2016ab, 2017) the function

$$
\begin{array}{rlrl}
f(\mathrm{~g}) & =\frac{1-\mathrm{e}^{-\mathrm{g} / \mathrm{g}_{01}}}{\left(\mathrm{~g} / \mathrm{g}_{01}\right)} & & \\
& =1-\frac{1}{2}\left(\frac{\mathrm{g}}{\mathrm{g}_{01}}\right)+\ldots \quad \text { for } \mathrm{g} \ll \mathrm{g}_{01}
\end{array}
$$

was used, while in Penner (2018) the function

$$
\begin{array}{rlrl}
f(\mathrm{~g}) & =e^{-\mathrm{g} / \mathrm{g}_{02}} & & \\
& =1-\left(\frac{\mathrm{g}}{\mathrm{g}_{02}}\right)+\ldots \quad \text { for } \mathrm{g} \ll \mathrm{g}_{02}
\end{array}
$$

was used. By (11), (12b), and (13b) the values for $\mathrm{g}_{01}$ and $\mathrm{g}_{02}$ are respectively

$$
\begin{aligned}
& \mathrm{g}_{01}=8.0 \times 10^{-11} \mathrm{~m} \mathrm{~s}^{-2} \\
& \mathrm{~g}_{02}=1.6 \times 10^{-10} \mathrm{~m} \mathrm{~s}^{-2} .
\end{aligned}
$$

Both (12a) and (13a) were used to determine the rotational curve of the galaxy, the rotational curves of spiral galaxies in general, the dynamics of the Coma cluster, the dynamics of the universe as a whole, and the dynamics of binary galaxies. As was stated in the introduction, this resulted in very good agreement with the observations. These observations fell within the weak field limit. So, whether (12a) or (13a) was used had only a minor effect on the results. In the case of strong gravitational fields where $g>g_{0}$, the differences between these two functions lead to very different results. Equation (12a) leads to the contribution to the gravitational field by the mass dipoles saturating (indeed this was the reason for initially picking this function) at a value of

$$
\mathrm{g}_{\mathrm{V}}=\mathrm{g}_{01}=8.0 \times 10^{-11} \mathrm{~m} \mathrm{~s}^{-2} .
$$

This corresponds to an equivalent mass within the orbit of Saturn, i.e. at 10 AU, of

$$
\Delta \mathrm{M}=1.3 \times 10^{-6} \mathrm{M}_{\odot}
$$

which greatly exceeds the value that is given by Pitjev \& Petjeva. As such, (12a) it is not a valid candidate for $f(\mathrm{~g})$. At a distance of $\mathrm{r}=10 \mathrm{AU}$, (13a) leads to a value of

$$
\Delta \mathrm{M}<<10^{-100} \mathrm{M}_{\odot}
$$

which is well within Pitjev \& Petjeva's value.

Although (13a) is a valid candidate for $f(\mathrm{~g})$, this manuscript will consider instead the following function which has a less extreme dependence on $\mathrm{g}$;

$$
f(g)=\left(1+\frac{g}{g_{02}}\right)^{-2}
$$




$$
\cong 1-2\left(\frac{\mathrm{g}}{\mathrm{g}_{02}}\right) \quad \text { for } \mathrm{g}<<\mathrm{g}_{02}
$$

which by (11) leads to

$$
\mathrm{g}_{02}=3.2 \times 10^{-10} \mathrm{~m} \mathrm{~s}^{-2} \text {. }
$$

This function leads to a value at a distance of $10 \mathrm{AU}$ of

$$
\Delta \mathrm{M}=2.92 \times 10^{-11} \mathrm{M}_{\odot}
$$

which also is well within Pitjev \& Petjeva's value. Using the expression for $f(\mathrm{~g})$ as given by (18a) then, from (5), leads to the following expression for $\mathbf{P}_{\mathbf{G}}$, the mass dipole moment density;

$$
\mathbf{P}_{\mathbf{G}}=-\frac{1}{4 \pi \mathrm{G}}\left(1+\frac{\mathrm{g}}{\mathrm{g}_{02}}\right)^{-2} \mathbf{g} \text {. }
$$

\subsection{Gravitational field around an isolated solar mass}

Given (21) for the mass dipole moment density, the equivalent mass density around any baryonic mass is determined by (2). The gravitational field surrounding any baryonic mass distribution can then be determined recursively using (3-4) with the initial guess being that the gravitational field is due solely to the baryonic mass. In the case of an isolated Sun being the baryonic mass of interest, the cross-section of the determined mass density due to the surrounding dipole distribution is shown in Figure 1a) and the mass density profile along the $\mathrm{z}$ axis is shown in Figure 2a). As is seen with these figures, the mass density due to the dipole distribution rises steadily, reaching a maximum near $6 \times 10^{-16} \mathrm{~kg} \mathrm{~m}^{-3}$ at approximately $2 \mathrm{kAU}$ and then steadily decreasing to approximately $3 \times 10^{-17} \mathrm{~kg} \mathrm{~m}^{-3}$ at $15 \mathrm{kAU}$. This density distribution is quite different from dark matter distributions where the distribution decreases steadily away from the baryonic centre. The cross-section of the resulting total gravitational field surrounding the isolated Sun in turn is shown in Figure 3a). Figure 4 shows the dependence that the resulting gravitational field has on distance from the Sun, due both solely to the solar mass itself and to the isolated solar mass with gravitational anti-screening. As is seen with Figure 4, within 2 kAU the gravitational field due solely to the baryonic mass of the Sun dominates. However, beyond $2 \mathrm{kAU}$ the effect of gravitational anti-screening becomes increasingly significant. While the field due solely to the solar mass falls off as $\mathrm{r}^{-2}$, that with the gravitational anti-screening added begins to fall off as $\mathrm{r}^{-1}$. As is found with galaxies, in the outer regions the enclosed mass will therefore be directly proportional to the distance to the baryonic mass centre which thereby will lead to the BTFR. At 
a distance of $10 \mathrm{kAU}$ the contribution that the dipole distribution makes to the gravitational field in this case is equal to approximately 1 solar mass.

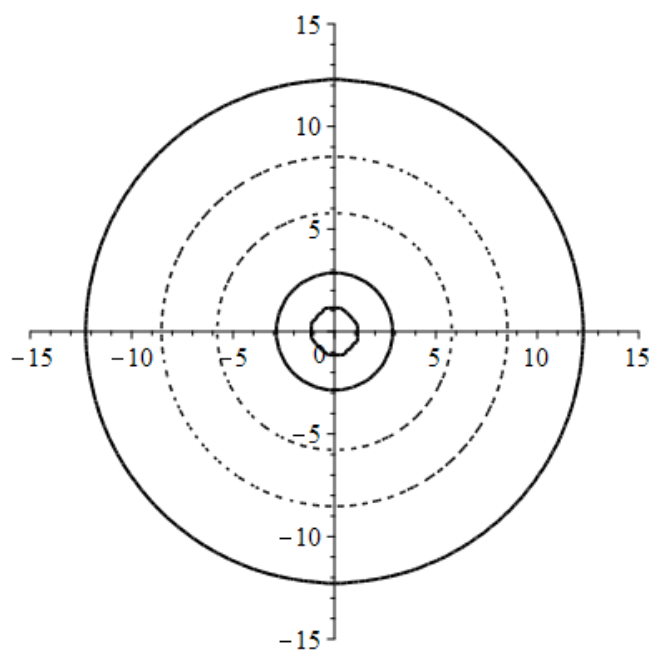

a)

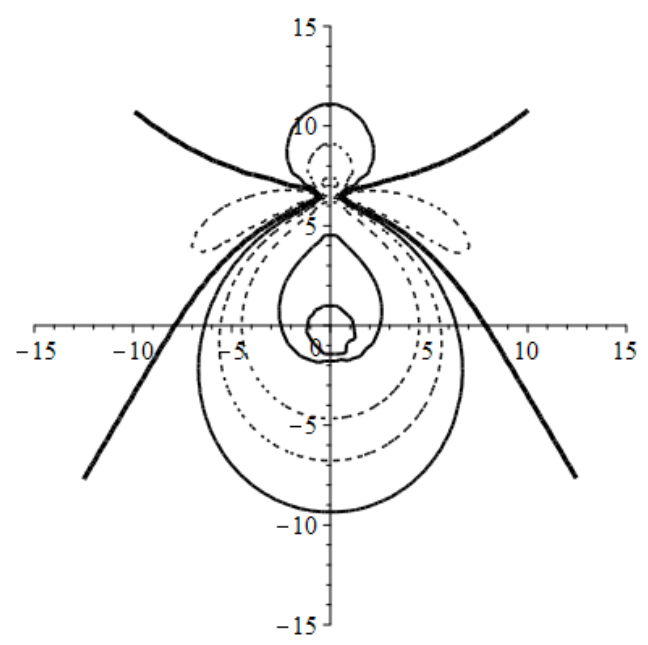

b)

Figure 1: Equivalent mass density cross-section: a) surrounding an isolated solar mass; b) surrounding a solar mass in the galactic gravitational field with the galactic centre located in the $+\mathrm{z}$ direction. Outer solid line corresponds to a mass density of $5.0 \times 10^{-17} \mathrm{~kg} \mathrm{~m}^{-3}$, the two inner solid lines correspond to a mass density of $5.0 \times 10^{-16} \mathrm{~kg} \mathrm{~m}^{-3}$. In the case of $\mathrm{b}$ ) the heavy solid line corresponds to a mass density of 0 , and outside of this line the mass density, although relatively low in value, is actually negative. Units along the axes are in kAU.

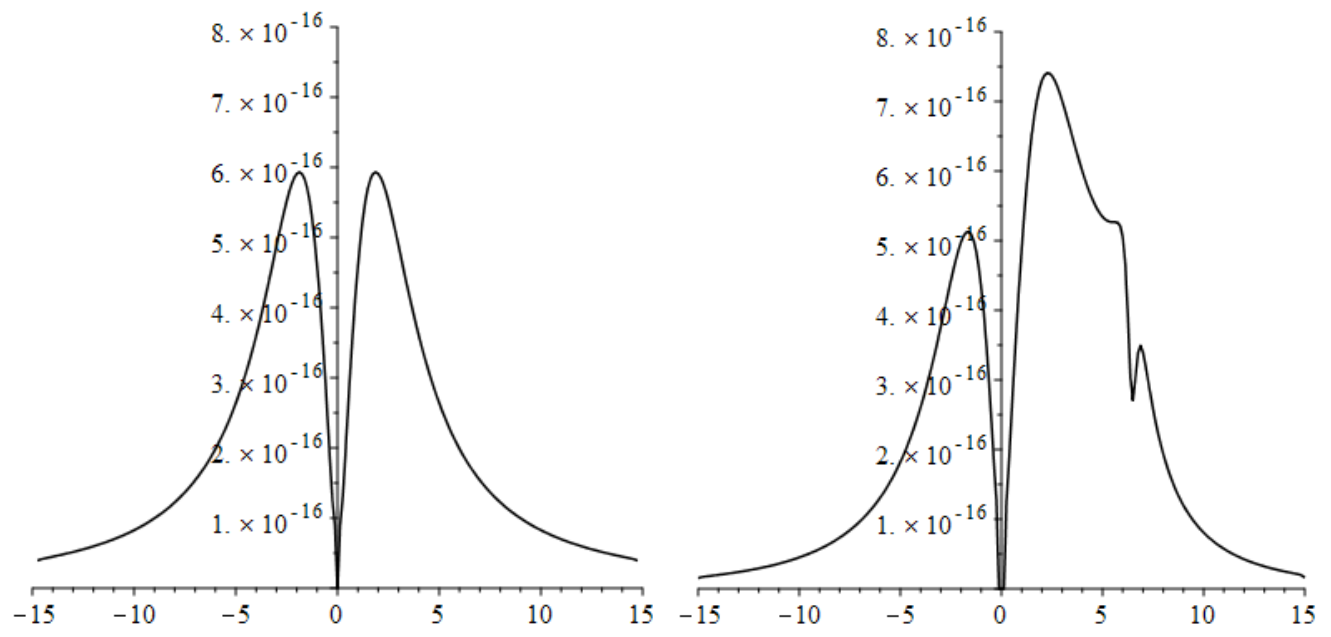

a)

b)

Figure 2: Equivalent mass density profile along the z-axis (vertical axis) for a) isolated solar mass; b) solar mass in the galactic gravitational field. In the case of b) the galactic centre is in the $+\mathrm{z}$ direction. Horizontal units are $\mathrm{kAU}$ and vertical units are $\mathrm{kg} \mathrm{m}^{-3}$. The kink at $\sim 6 \mathrm{kAU}$ corresponds to the distance where the resulting field surrounding the Sun is equal in magnitude to the galactic field. 


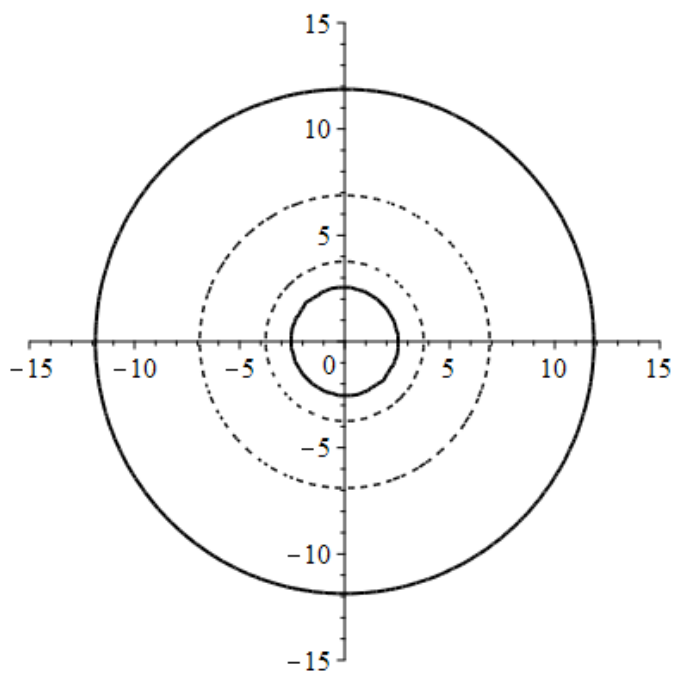

a)

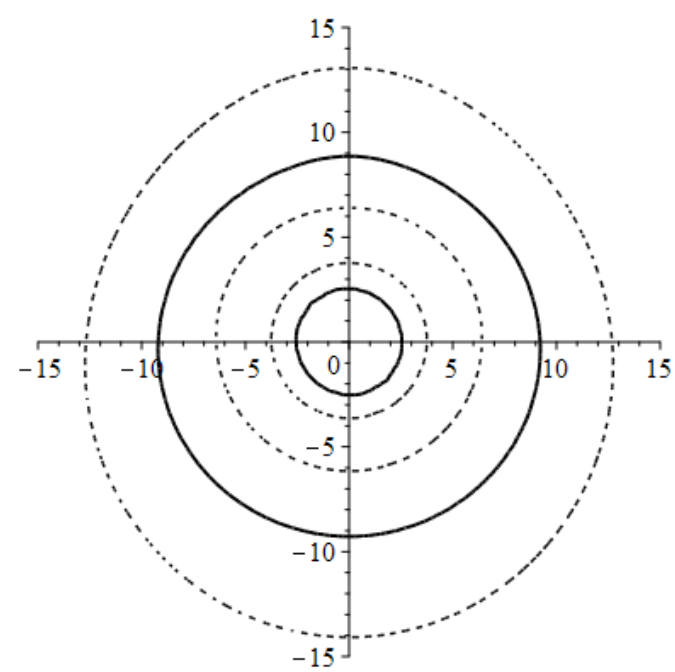

b)

Figure 3: Cross-section of the magnitude of the gravitational field surrounding: a) an isolated solar mass; b) the solar mass in the galactic gravitational field. In the case of b) the constant galactic gravitational field has been subtracted out. The outer solid line has a value of $1.0 \times 10^{-10}$ $\mathrm{m} \mathrm{s}^{-2}$ and the inner solid line has a value of $1.0 \times 10^{-9} \mathrm{~m} \mathrm{~s}^{-2}$. Units along the axes are in kAU.

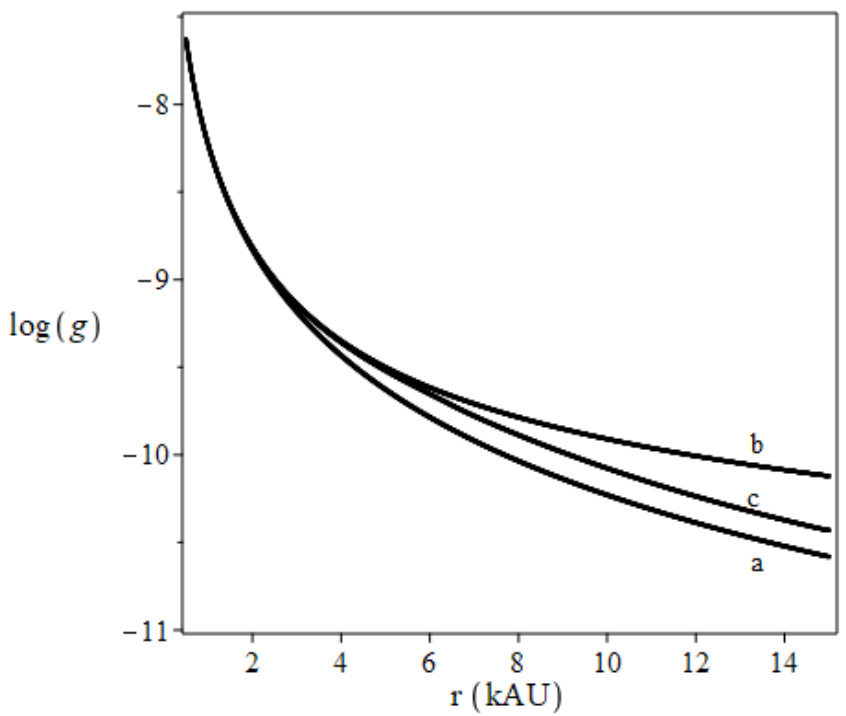

Figure 4: The magnitude of the gravitational field in the cases of: a) no gravitational antiscreening, (b) isolated solar mass with gravitational anti-screening, (c) solar mass in the galactic field with gravitational anti-screening. 


\subsection{Inclusion of the Galactic gravitational field}

The theory of gravitational anti-screening is a non-linear theory, specifically with regards to the dependence that $\mathbf{P}_{\mathbf{G}}$ has on $\mathbf{g}$ and the resulting dependence that $\mathrm{g}_{\mathrm{V}}$ then has on $\mathrm{g}$. The value of the external gravitational field due to the Galaxy at the location of the Sun is approximately $2.0 \times 10^{-10} \mathrm{~m} \mathrm{~s}^{-2}$. This is of the order of the gravitational field due to the Sun at a distance of $5 \mathrm{kAU}$, and therefore the external galactic field will have a significant effect on the results. A similar issue arises in other non-linear theories such as MOND. In this context M. Milgrom (1986) considered the effect of a point mass, such as the Sun, in a constant external field. Banik and Zhao (2018a) also considered the solution to the MOND equations in the case of a point mass immersed in a constant gravitational field.

In order to determine the effect that the external galactic gravitational field has on the mass density distribution surrounding the Sun, it is approximated that the galactic field is constant within a distance of $15 \mathrm{kAU}$ from the Sun. The resulting cross-section of the mass density distribution surrounding the Sun within this constant gravitational field is shown on Figure 1b) and the mass density profile along the $\mathrm{z}$-axis is shown on Figure $2 \mathrm{~b}$ ). The galactic centre lies in the $+\mathrm{z}$ direction for both of these figures. For this calculation it is approximated that the contribution that the mass density distribution beyond $15 \mathrm{kAU}$ makes to the field within $15 \mathrm{kAU}$ can be neglected. As seen in Figure 1b) the galactic gravitational field has a very strong impact on the resulting mass density distribution. Along the z-axis, i.e. Figure $2 b$ ), the impact is fairly straight forward to understand. In the $+\mathrm{z}$ direction the galactic field opposes the solar field, leading to a reduced value of $g$ and thereby larger values for both $\mathbf{P}_{\mathbf{G}}$ and $\rho \mathrm{v}$. In the $-\mathrm{z}$ direction the galactic field is in the same direction as the solar field, leading to an increased value of $g$ and resulting smaller values for both $\mathbf{P}_{\mathbf{G}}$ and $\rho_{\mathrm{V}}$. It is when one moves away from the z-axis that things become much more complicated, as can be seen with Figure 1b). It is not just that $\mathbf{P}_{\mathbf{G}}$ becomes more complex, the mass density itself depends on the divergence of $\mathbf{P}_{\mathbf{G}}$.

Figure $3 b$ ) shows the cross-section of the resulting total gravitational field surrounding the Sun. The constant galactic gravitational field has been subtracted out given that the Sun and the mass dipoles are in free fall about the galactic centre. The resulting gravitational field is no longer perfectly spherically symmetric, although not too far off. Figure 4 includes the effect that the external galactic gravitational field has on average gravitational field surrounding the Sun. As is 
seen, beyond $5 \mathrm{kAU}$ the external galactic field begins to play a significant role, with the gravitational field going from its $\mathrm{r}^{-1}$ dependence, for an isolated solar mass with gravitational antiscreening, back to falling off as $\mathrm{r}^{-2}$. The effect of the external galactic field therefore significantly reduces the effect of gravitational anti-screening. The net effect is that beyond $5 \mathrm{kAU}$ the overall contribution that the dipole distribution makes to the solar gravitational field corresponds to the solar mass being approximately 1.4 greater.

\subsection{Effect on orbital parameters}

\subsubsection{Aphelia}

The greatest impact that the resulting larger gravitational field will have on the aphelion of orbiting bodies will be on those bodies which have high eccentricities along with an aphelion greater than $2 \mathrm{kAU}$. This is the realm of long-period comets. The aphelia of these bodies are very sensitive to changes in the gravitational field.

With or without gravitational anti-screening, within $2 \mathrm{kAU}$ of the Sun the gravitational field due to the Sun will dominate. In this case, the specific orbital energy of an object will be given by

$$
\frac{1}{2} v_{p}^{2}-\frac{G M_{\odot}}{r_{p}}=-\frac{G M_{\odot}}{2 a}
$$

where (a) is the determined semi-major axis based on current gravitational theory, $r_{p}$ is the perihelion distance $(<2 \mathrm{kAU})$, and $\mathrm{v}_{\mathrm{p}}$ is the object's speed at perihelion. In the case of bound orbits, the value of (a) will be positive, while for unbound orbits its value will be negative. In the case of highly eccentric bound orbitals, i.e. where e $\cong 1.0$, the objects speed at perihelion will be much greater than the objects speed at aphelion. It then also follows that

$$
\frac{1}{2} \mathrm{v}_{\mathrm{p}}^{2}=\int_{\mathrm{r}_{\mathrm{p}}}^{\mathrm{r}_{\mathrm{a}}} \mathrm{g} \mathrm{dr}
$$

where $r_{a}$ is the aphelion distance. Therefore by (22) and (23)

$$
\mathrm{GM} \odot\left(\frac{1}{\mathrm{r}_{\mathrm{p}}}-\frac{1}{2 \mathrm{a}}\right)=\int_{\mathrm{r}_{\mathrm{p}}}^{\mathrm{r}_{\mathrm{a}}} \mathrm{g} \mathrm{dr}
$$

In the case where there is no gravitational anti-screening, and the gravitational field is dominated by the Sun, the aphelia for these highly eccentric orbits will be equal to $2 \mathrm{a}$. In the case where there 
is gravitational anti-screening, to determine the aphelia the value of $\mathrm{g}$, as determined from the model and as shown in Figure 4, is used. For calculation purposes, (24) can be generalized to

$$
\mathrm{GM} \odot\left(\frac{1}{r_{o}}-\frac{1}{2 a}\right)=\int_{r_{o}}^{r_{a}} g d r
$$

with $r_{o}$, where $r_{o}<2 \mathrm{kAU}$, being a reference distance within which the gravitational field due to the Sun dominates.

Figure 5 compares the aphelia as determined from the theory to the case where there is no gravitational anti-screening and the aphelia are just equal to $2 \mathrm{a}$. As is seen in the Figure 5, for these high eccentric orbits the differences between the aphelia determined from the theory of gravitational anti-screening and those determined for no anti-screening become very large for aphelia which exceed $5 \mathrm{kAU}$. These large differences will lead to definitive predictions regarding the aphelia of long-period comets.

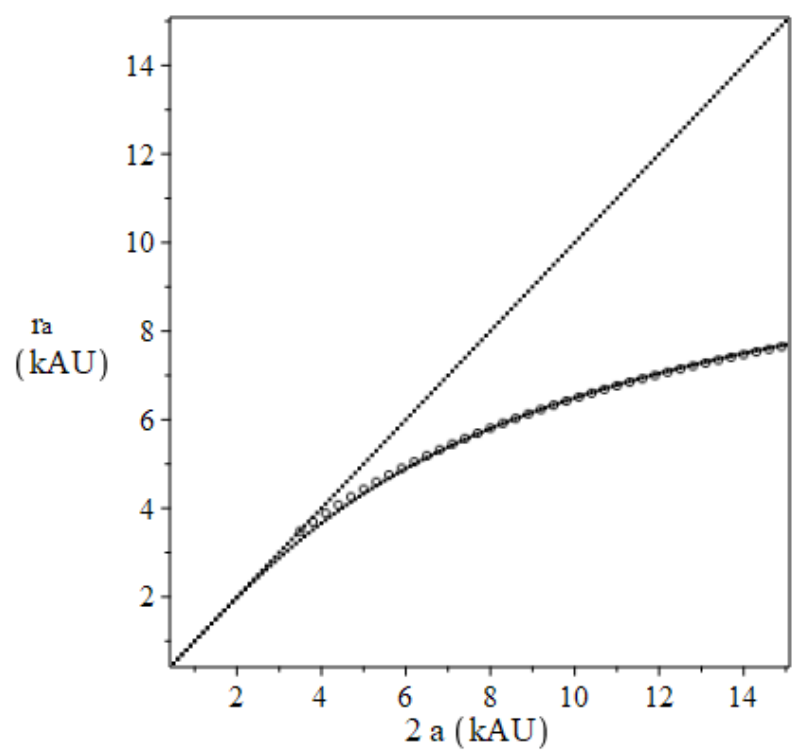

Figure 5: The determined aphelion distances versus 2a. The straight line is the no gravitational anti-screening case where the aphelion distances are equal to $2 \mathrm{a}$. The values given by o are the values as determined from (29).

To get a better understanding of how the greater gravitational field effects the aphelia of long-period comets, the calculated gravitational field as shown in Figure $4 \mathrm{c}$ will be approximated by 


$$
\begin{array}{ll}
\mathrm{g}=\frac{\mathrm{G} \mathrm{M}_{\odot}}{\mathrm{r}^{2}} & \mathrm{r} \leq 3.45 \mathrm{kAU}, \text { and } \\
\mathrm{g}=\frac{\mathrm{G}\left(1.4 \mathrm{M}_{\odot}\right)}{\mathrm{r}^{2}} & \mathrm{r}>3.45 \mathrm{kAU} .
\end{array}
$$

The specific orbital energy of a comet will therefore jump from

$$
\frac{1}{2} \mathrm{v}_{3.45}{ }^{2}-\frac{\mathrm{G} \mathrm{M}_{\odot}}{\mathrm{r}_{3.45}}=-\frac{\mathrm{G} \mathrm{M}_{\odot}}{2 \mathrm{a}},
$$

where $r_{3.45}=3.45 \mathrm{kAU}$ and $\mathrm{v}_{3.45}$ is the comet's speed at $3.45 \mathrm{kAU}$, to

$$
\frac{1}{2} \mathrm{v}_{3.45}{ }^{2}-\frac{\mathrm{G}\left(1.4 \mathrm{M}_{\odot}\right)}{\mathrm{r}_{3.45}}=-\frac{\mathrm{G}\left(1.4 \mathrm{M}_{\odot}\right)}{\mathrm{a}_{1}}
$$

as the comet passes the $3.45 \mathrm{kAU}$ mark. Equations (27) and (28) then lead to the following approximate relationship between $\mathrm{r}_{\mathrm{a}}=2 \mathrm{a}_{1}$, the aphelia with gravitational anti-screening, and $2 \mathrm{a}$, the aphelia that falls out of current gravitational theory;

$$
r_{a}=1.4\left(\frac{0.4}{r_{3.45}}+\frac{1}{2 a}\right)^{-1} \text {. }
$$

The dependence that $r_{a}$ has on $2 a$, as given by (29), is included in Figure 5. As is seen in the figure (29) provides a very good approximation to the actual results.

In the case where the orbits are circular, or nearly so, the differences between the orbital speeds of distant bodies, in the cases of having or not having gravitational anti-screening effects, although not as dramatic as the aphelia differences are still significant. Figure 6 shows the results. At orbital radii > $5 \mathrm{kAU}$ the orbital speeds in the case of gravitational anti-screening are approximately $20 \%$ greater than what is currently predicted by gravitational theory. If the Sun was isolated, i.e. no galactic field effects, the orbital speeds would approach the BTFR value for the Sun of $382 \mathrm{~m} / \mathrm{s}$, found by substituting $\mathrm{M}_{\mathrm{B}}=1 \mathrm{M}_{\odot}$ into (1). The $20 \%$ greater orbital speeds at these distances is the same as that found by applying MOND to wide binary stars separated by $\geq 3 \mathrm{kAU}$ (Banik\&Zhao 2018b).

\subsubsection{Perihelion precession}

The impact that dark matter within the solar system would have on the perihelion drift of orbiting objects has previously been considered (Pitjev \& Petjeva 2013, Khriplovich 2007). The resulting perihelion shift per orbital period for near circular orbits, i.e. $\mathrm{e} \cong 0$, is given by

$$
\Delta \theta_{0}=-4 \pi^{2} \rho \mathrm{DM}(\mathrm{r}) \mathrm{r}^{3} / \mathrm{M}_{\odot}
$$




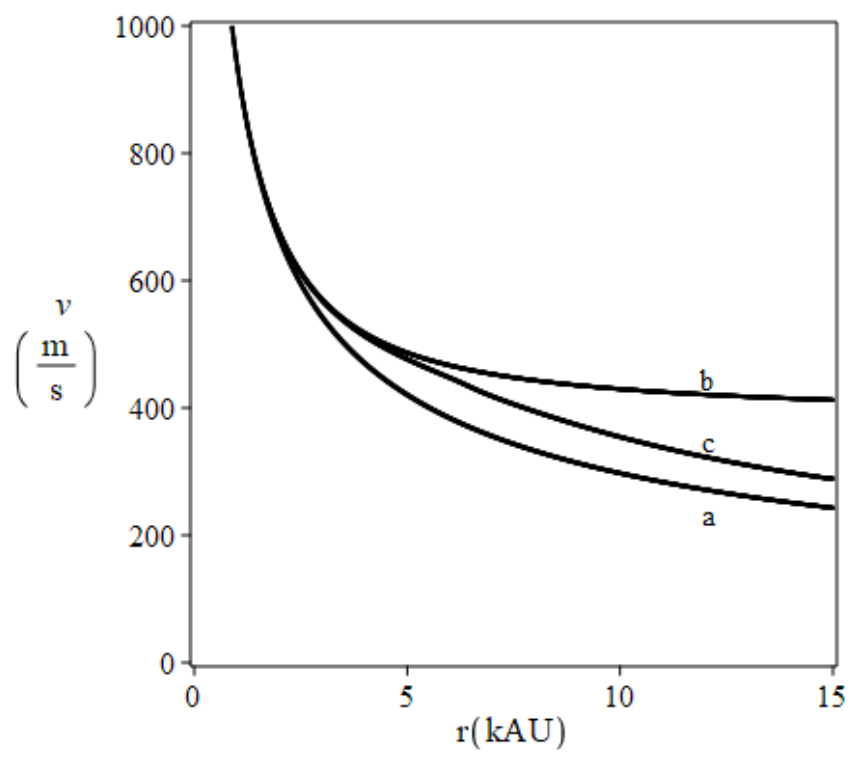

Figure 6: The orbital speed versus distance from the Sun in the cases of: a) no gravitational anti-screening, (b) isolated solar mass with gravitational anti-screening, (c) solar mass in the galactic field with gravitational anti-screening.

where $r$ is the radius of the orbit and $\rho_{\mathrm{DM}}(\mathrm{r})$ is the density of the dark matter, taken to be spherically symmetric about the Sun, at that position. Given that gravitational anti-screening itself leads to a mass density distribution surrounding the Sun, these same results can be used by substituting $\rho v(r)$ for $\rho_{\mathrm{DM}}(\mathrm{r})$ in $(30)$.

For the given model for $f(\mathrm{~g})$, in the case where $\mathrm{g} \gg \mathrm{g}_{0}$, it follows that

$$
f(\mathrm{~g}) \cong \mathrm{g}_{0}{ }^{2} / \mathrm{g}^{2}
$$

which from (6) then leads to

$$
\begin{aligned}
\mathrm{g}_{\mathrm{V}} & =\mathrm{g}_{0}{ }^{2} / \mathrm{g} \\
& =\frac{\mathrm{g}_{0}{ }^{2}}{\mathrm{GM}_{\odot}} \mathrm{r}^{2} .
\end{aligned}
$$

Equation (33) in turn results in

$$
\rho v=\frac{g_{0}^{2}}{\pi G^{2} M_{\odot}} r .
$$

Substituting (34) into (30) then leads to the following perihelion shift per period for the given model;

$$
\begin{aligned}
\Delta \theta_{0} & =\frac{4 \pi g_{0}^{2}}{\mathrm{G}^{2} \mathrm{M}_{\odot}^{2}} \mathrm{r}^{4} \\
& =-\left(7.62 \times 10^{-6}\right) \mathrm{r}^{4} \text { mas/period }
\end{aligned}
$$


where the orbital distance $r$ is in units of AU.

\section{Results}

\subsection{Aphelia of long-period comets}

The source of long-period comets is generally taken to be what is known as the Oort cloud; whose existence was first proposed by Oort to explain the energy distribution of long-period comets. In current gravitational theory these orbital energies correspond to aphelia ranging from between $~ 1 \mathrm{kAU}$ and $~ 1000 \mathrm{kAU}$.

Krolikowska (2014) has provided the orbital elements for 119 of these long-period comets. Their orbits were determined from positional data with dynamical calculations performed both backward and forward up to 250 AU from the Sun, so as to include planetary perturbations. The determined eccentricity for all of these comets is e $>0.9994$, with five of the comets having e $>1$. In the case of no gravitational anti-screening, these 5 comets are therefore unbound. Based on current gravitational theory, the 114 bound comets have determined semi-major axes ranging from 6.7 kAU to $478 \mathrm{kAU}$ and corresponding aphelia between $13 \mathrm{kAU}$ and $957 \mathrm{kAU}$. The distribution of the aphelia for the 111 of these comets whose aphelion is below $300 \mathrm{kAU}$, is shown in Figure 7. For these long-period comets, there is a broad peak of aphelia between $10 \mathrm{kAU}$ and $100 \mathrm{kAU}$, with the peak at around $50 \mathrm{kAU}$. The results from Section 2 will now be applied to this set of 119 long-period comets.

As can be seen in Figure 5, at values where $2 \mathrm{a}>5 \mathrm{kAU}$ the gravitational anti-screening theory leads to aphelia that are much smaller than those predicted by current gravitational theory. The distribution of aphelia calculated, using (18a) for $f(\mathrm{~g})$, for all 119 comets (the 5 comets which were initially unbound are now bound) are shown in Figure 8. The peak of the aphelia is now at only approximately $10 \mathrm{kAU}$. In addition, the range of aphelia is also much narrower than that without gravitational anti-screening. Although these results are for a particular $f(\mathrm{~g})$, the specific function $f(\mathrm{~g})$ used in the model will have only secondary effects on these results.

The differences between the predicted aphelia in the case of no gravitational anti-screening and the predicted aphelia with gravitational anti-screening are very large. The results indicate that the source of this representative set of long-period comets is from a cloud of comets that occupies a quite narrow range of distances centred at approximately 10 kAU from the Sun rather than the very broad range of distances centred at approximately $50 \mathrm{kAU}$ that falls out from current 
gravitational theory. In addition, from Figure 6, at these distances of (10 \pm 2$)$ kAU the comets in this cloud will have orbital speeds approximately $20 \%$ greater than the speeds that would be determined from current gravitational theory.

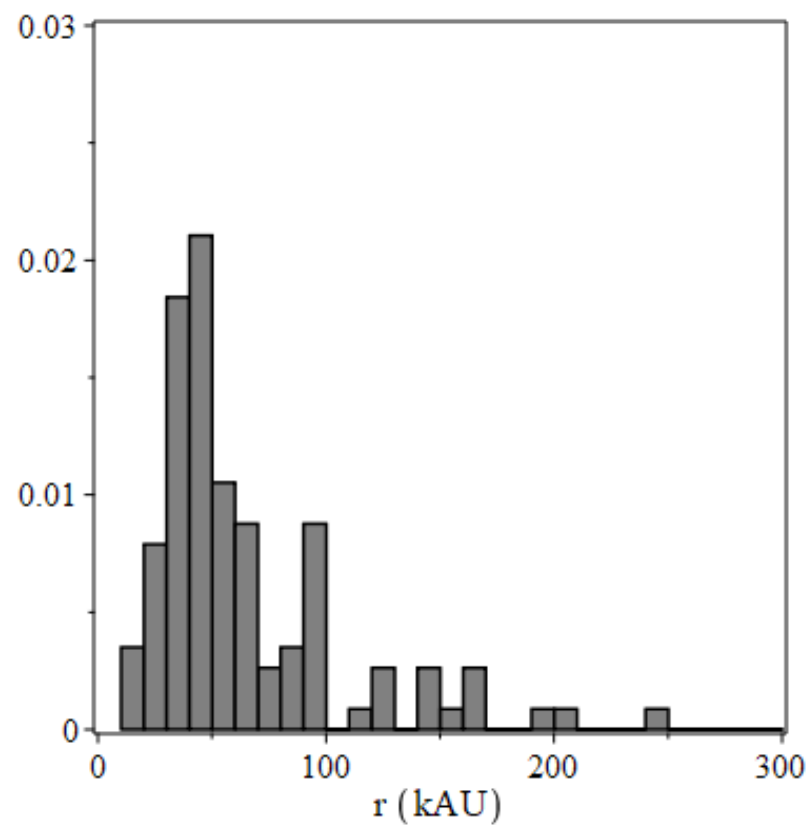

Figure 7: Histogram of aphelia for the 111 bound comets listed in Krolikowska (2014) whose aphelia are $<300 \mathrm{kAU}$.

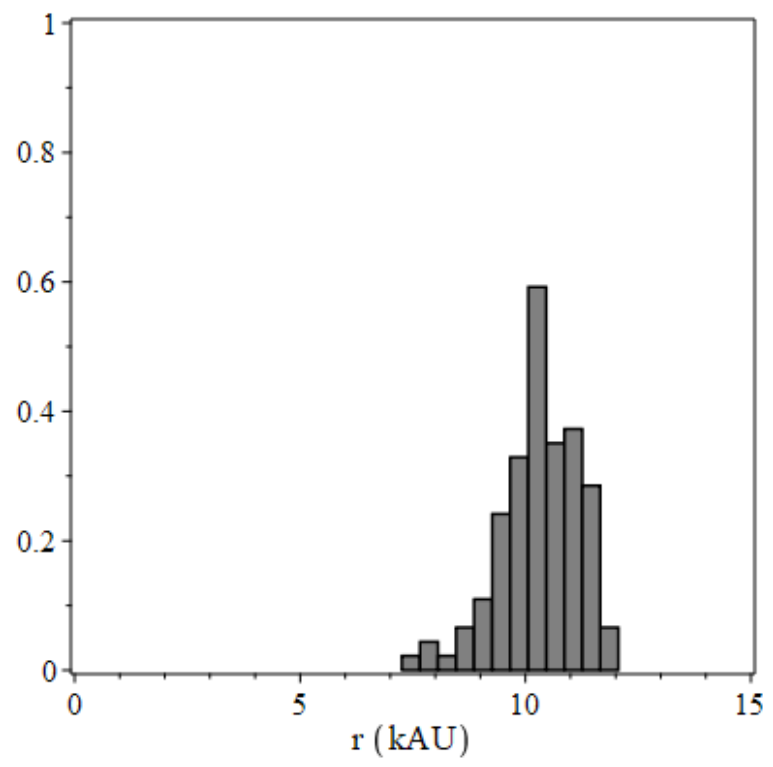

Figure 8: Histogram of aphelia for all of the 119 comets listed in Krolikowska (2014) in the case of gravitational anti-screening using the $f(\mathrm{~g})$ as given by (18a). 


\subsection{Perihelion shift of the planets}

Using (35b) the additional perihelion shift for the planets of the solar system due to gravitational anti-screening can be determined. The results are shown in Table 1 along with the observational constraints as determined by Pitjev\&Pitjeva (2013) for the inner six planets. Also included in the table are predicted values for orbiting bodies at distances of $100 \mathrm{AU}$ and $500 \mathrm{AU}$.

\begin{tabular}{|l|l|l|l|c|c|}
\hline Planet & $\begin{array}{c}\mathrm{a} \\
(\mathrm{AU})\end{array}$ & $\begin{array}{l}\text { Period } \\
(\mathrm{yrs})\end{array}$ & $\begin{array}{c}\Delta \theta_{0} \\
(\mathrm{mas} / \text { period })\end{array}$ & $\begin{array}{c}\text { anti-screening } \\
\text { Precession rate } \\
\left(\mathrm{mas} \mathrm{cy}^{-1}\right)\end{array}$ & $\begin{array}{c}\text { Observations } \\
\text { Pitjev\&Pitjeva } \\
\left(\mathrm{mas} \mathrm{cy}^{-1}\right)\end{array}$ \\
\hline Mercury & 0.3871 & 0.2408 & $-1.71 \times 10^{-7}$ & $-7.11 \times 10^{-5}$ & $-(2.0 \pm 3.0)$ \\
\hline Venus & 0.7233 & 0.6152 & $-2.09 \times 10^{-6}$ & $-3.40 \times 10^{-4}$ & $(2.6 \pm 1.6)$ \\
\hline Earth & 1.0000 & 1.0000 & $-7.62 \times 10^{-6}$ & $-7.62 \times 10^{-4}$ & $(1.9 \pm 1.9)$ \\
\hline Mars & 1.5273 & 1.8809 & $-4.15 \times 10^{-5}$ & -0.00220 & $-(0.020 \pm 0.037)$ \\
\hline Jupiter & 5.2028 & 11.862 & -0.00558 & -0.0471 & $(58.7 \pm 28.3)$ \\
\hline Saturn & 9.5388 & 29.458 & -0.0631 & -0.214 & $-(0.32 \pm 0.47)$ \\
\hline Uranus & 19.1914 & 84.01 & -1.03 & -1.23 & \\
\hline Neptune & 30.0611 & 164.79 & -6.22 & -3.78 & \\
\hline & 100 & 1000 & -762 & -76.2 & \\
\hline & 500 & 11,180 & $4.76 \times 10^{5}$ & -4260 & \\
\hline
\end{tabular}

Table 1: Predicted additional precision rates and observational constraints as given by Pitjev\&Pitjeva (2013).

In Table 1 the uncertainties in the observational constraints are in general much greater than the predicted additional precession rate. The greatest discrepancy between prediction and constraint is with Jupiter. However, it must be pointed out that this discrepancy also exists with current gravitational theory as an anomalous precession rate of zero is predicted for all orbiting bodies. A key feature of the predicted precession rates is that they increase rapidly with orbital radius. This indicates that radio tracking of a satellite orbiting around the most outermost planets would seem to be the best path to follow with regards to testing the theory of gravitational antiscreening as well as other alternative theories to dark matter. 


\section{Conclusion}

The model of mass dipoles presented, i.e. massless particles with an intrinsic mass dipole moment, is compatible with general relativity in that such particles would free fall in a gravitational field. In addition, such a mass dipole eliminates the need for a particle with one or more of its active gravitational, passive gravitational, or inertial masses negative. Given that these mass dipoles will not naturally align in a gravitational field led to the realization that a given mass must itself be a source of these dipoles and that these dipoles come into existence with their dipole moment already aligned with the gravitational field. The mechanism of virtual gravitons dissociating into these mass dipoles is attractive in the symmetry between the screening of electric charge by virtual electric dipoles in QED and the anti-screening of mass by virtual mass dipoles. It would seem to indicate that the astronomical observations attributed to dark matter are actually a quantum gravity effect.

As demonstrated in the manuscript, gravitational anti-screening leads to a contribution to the gravitational field within the solar system which becomes significant for distances beyond a few kAU from the Sun. This is the realm of long-period comets. It is found that gravitational antiscreening, and the resulting increased gravitational field, will greatly reduce the aphelia of these comets and increase their orbital speeds. If it is taken that the source of these comets is the Oort cloud then the Oort cloud is much closer and much narrower in range than is currently conjectured and the orbital speeds of the comets in the cloud are greater. To summarize a key result from this manuscript: It is predicted from the theory of gravitational anti-screening that a large cluster of cometary bodies will be found at a distance of (10 \pm 2$)$ kau from the Sun traveling at these distances with orbital speeds approximately $20 \%$ greater than that calculated from current gravitational theory.

Although, currently observations of comet size bodies at distances of $10 \mathrm{kAU}$ from the Sun are not possible, such measurements may be possible in the not so distant future. The Whipple mission (Alcock) proposes to search for members of the Oort cloud by the occultation of stars. This would be undertaken by a small telescope in space. A primary goal of the mission would be to provide estimates of the size and number of cometary bodies at these great distances. However, analysis of the diffraction fringes will also allow for rough estimates of the distances to the occulting objects. At the moment, the Whipple mission is just a proposal and is designed only to be able to make observations out to distance of $3 \mathrm{kAU}$, however, it does show that measurements 
at such great distances are feasible. Future projects like the Whipple mission, can therefore do much more than estimate the number and size of cometary bodies at these great distances. By determining comet distances and orbital speeds, they have the potential to decide between dark matter and alternate theories such as gravitational anti-screening.

The theory of gravitational anti-screening also leads to an additional precession of orbiting bodies. It is found that the precession rate of orbiting bodies due to gravitational anti-screening increases rapidly with orbiting distance. At present the uncertainties in the constraints are much greater than the predicted precession rates. However, future measurements of the orbital parameters of the most distant solar system bodies would seem to be the path to follow.

Although the specific values predicted in this manuscript are based on the particular function used for $f(\mathrm{~g})$, the general results will hold. Gravitational anti-screening will lead to significantly smaller aphelia for long-period comets and an additional precession rate which increases with distance for solar system bodies.

\section{References:}

Alcock, C., Holman, M., Lehner, M., et al.: https://solarsystem.nasa.gov/studies/37/whippleexploring-the-solar-system-beyond-neptune-using-a-survey-for-occultations-of-bright-stars/ Banik, I.,Zhao, H.:arXiv:1509.08457 (2018a)

Banik, I.,Zhao, H.: MNRAS 480, 2660, (2018b)

Blanchet, L.: Class. Quant. Grav. 24, 3529, astro-ph/0605637 (2007a)

Blanchet, L.: Class. Quant. Grav. 24, 3541, gr-qc/0609121 (2007b)

Blanchet, L.: LeTiec, A. arXiv.org/abs/0804.3518 (2008)

Bondi, H.: Rev. Mod. Phys. 29(3), 423 (1957)

Hajdukovic, D.S.: Astrophys. Space Sci. 334, 215 (2011a)

Hajdukovic, D.S.: Adv. Astron. 196, 852 (2011b)

Hajdukovic, D.S.: Mod. Phys. Lett. A 26, 1555 (2011c)

Hajdukovic, D.S.: Astrophys. Space Sci. 337, 9 (2012a)

Hajdukovic, D.S.: Astrophys. Space Sci. 339, 1 (2012b)

Hees, A., Folkner, W.M., Jacobson, R.A., Park, R.S.:arXiv:1402.6950v2[gr-qc] (2014)

Khriplovich, I.B.: Int. J. Modern Physics D 16(09) 1475 (2007)

Krolikowska, M.: arXiv:1407.0553v1 (2014)

McGaugh, S.S.: Astron. J 143, 40 (2012)

Milgrom, M.:Astrophys. Jour. 302, 617 (1986)

Penner, A.R.: Astrophys. Space Sci. 361, 124 (2016a)

Penner, A.R.: Astrophys. Space Sci. 361, 361 (2016b)

Penner, A.R.: Astrophys. Space Sci. 362:80 (2017)

Penner, A.R.: Gravitational Anti-Screening as an Alternative to Dark Matter. In: Mehler, N. (ed.) Research Advances in Astronomy. pp 1-48. Nova US, New York, NY (2018)

Pitjev, N.P., Pitjeva, E.V.: Astron. Lett., 39-3, 141 (2013) 